\title{
Los retos de la explotación masiva de datos administrativos para la investigación y las políticas públicas: una visión desde dentro de la Administración
}

\section{The challenges of the massive use of administrative data for research and public policy: a view from inside the public administration}

\author{
Israel Arroyo \\ José Fernández Albertos \\ Ministerio de Inclusión, Seguridad Social y Migraciones
}

\section{Resumen}

Con el fin de contribuir al debate en torno a cómo corregir el déficit de explotación de datos administrativos para fines de investigación y evaluación de políticas públicas en el caso español, se analiza la cuestión desde la perspectiva de las administraciones que generan y gestionan esos datos, y se describen los principales obstáculos que están detrás de ese déficit. En la segunda parte del artículo se exponen una serie de cambios y reformas para revertirlos o sortearlos.

Palabras clave: datos administrativos, big data, registros administrativos, evaluación de políticas, análisis de políticas públicas

Códigos JEL: C80, D63.

\section{Abstract}

To contribute to the debate on how to correct for insufficient use of administrative data for research or policy evaluation in the Spanish case, we analyze the issue from the perspective of the administrations that produce and manage administrative records and describe the main obstacles that explain the deficit in its use. In the second part of the article we present a series of changes and reforms that could contribute circumventing these obstacles.

Keywords: administrative data, big data, administrative records, policy evaluation, public policy analysis 


\section{Introducción}

La revolución que ha supuesto la posibilidad de explotar de forma masiva y entrelazada grandes bases de datos para la investigación y el diseño de políticas públicas en las últimas décadas ha dotado de un nuevo valor al papel de la administración como creador y gestor de registros administrativos. De hecho, no es exagerado afirmar que el «descubrimiento» de las posibilidades que ofrecían estos registros ha sido una de las principales fuerzas que han impulsado esta revolución.

La explotación de datos administrativos para la investigación académica y la evaluación de políticas públicas ofrece ventajas que van más allá de las asociadas al poder estadístico que confieren su alto número de observaciones. Así, a diferencia de otras formas de recogida de datos, el mantenimiento de registros administrativos permite el análisis de la información procedente de colectivos de difícil acceso (por ejemplo, es bien conocida la dificultad de los estudios basados en encuestas de ingresos o gastos para capturar información en los dos extremos de la distribución de la renta), y la combinación de fuentes de información de naturaleza diversa que hasta hace poco era difícil de integrar dentro de un mismo análisis. Igualmente, la regularidad con la que por lo general se mantienen los registros administrativos permite además una explotación longitudinal de la información muy difícil de replicar con otro tipo de fuentes.

La puesta a disposición de estas bases de datos para finalidades académicas y de evaluación y diseño de políticas públicas no es sin embargo un proceso sencillo (Perrin et al., 2015). En última instancia, el problema consiste en que estos datos administrativos se han recogido para mantener un recuento de operaciones de la propia administración, y no tanto (o no principalmente) para impulsar el análisis y la evaluación de las políticas que la administración despliega. Eso explica que la mayor parte de países de nuestro entorno hayan tenido que crear una nueva arquitectura legal e institucional para permitir el uso reglado y seguro de estos datos. En España hemos ido con algo de retraso en el desarrollo de esa arquitectura, lo que hace que todavía hoy la existencia de registros administrativos, generados y alimentados durante décadas para finalidades de gestión, coexiste con una explotación limitada y parcial de los datos contenidos en ellos. Es importante que nuestro país no se quede atrás en este cambio, y tenemos la ventaja de que podemos aprender de las estrategias de aquellos países que lo han hecho antes ${ }^{1}$. Pero además de importar las mejores prácticas, es preciso conocer bien los obstáculos con los que esta revolución se enfrenta en nuestro contexto.

Afortunadamente, existe un creciente interés público en torno a esta cuestión, que ha dado pie a un vibrante conjunto de iniciativas y contribuciones que están poniéndolo en el centro de la agenda política (Almunia \& Rey-Biel, 2021; De la Fuente et al., 2021; AIReF, 2020). Con el fin de contribuir a este debate en torno a cómo corregir el déficit de explotación de datos en el caso español, en este artículo catalo-

\footnotetext{
${ }^{1}$ En AIReF (2020) o Brugarolas et al. (2020) se pueden encontrar algunos ejemplos.
} 
gamos lo que a nuestro juicio son los principales obstáculos que están detrás de ese déficit, y a continuación exponemos una serie de cambios y reformas para revertirlos o sortearlos. En este abanico de medidas incluimos actuaciones ya realizadas en el ámbito de la Seguridad Social, una de las principales instituciones estatales generadoras de datos en nuestro país, pero también intervenciones de naturaleza más amplia de medio plazo que a nuestro juicio serán esenciales para consolidar un nuevo marco institucional de explotación de datos administrativos para la investigación.

\section{La naturaleza multidimensional del problema: hacia una taxonomía de los obstáculos al uso de datos administrativos para la investigación}

A la hora de entender cuáles son los principales pasos a dar para incorporarnos a la primera línea en la utilización de datos procedentes de registros administrativos con fines de investigación, es preciso hacer un ejercicio de identificación de los obstáculos que todavía hoy persisten. Estos obstáculos son de naturaleza diversa, y van desde problemas de diseño institucional a la existencia de diferentes tipos de inercias en el seno de las Administraciones públicas. La principal conclusión de este repaso es que no existe una explicación sencilla y unívoca a la pregunta de por qué los datos administrativos españoles están hoy infrautilizados, y el corolario es que tampoco existe una «bala de plata» que solucione este problema. Necesitaremos diferentes tipos de reformas e intervenciones.

\subsection{El marco legal garantista y el problema de la confidencialidad y privacidad}

En España, la legislación que regula la producción y uso de registros administrativos es por lo general muy garantista, y como principio no contempla expresamente el uso de los mismos para fines distintos a los que determinan su creación y en particular para fines de investigación o de evaluación. A falta de modificaciones normativas, la utilización ha de ampararse en los casos, muy tasados, que la normativa general de protección de datos contempla para fines de investigación.

La legislación reconoce el derecho de acceso a la información administrativa con fines estadísticos, pero no para investigación aplicada, aunque la legislación comunitaria (Reglamento (UE) 2016/679) sí contempla la cesión de la información en poder de la administración para la investigación científica, y se promueve la apertura de documentos y datos de las Administraciones públicas (Directiva 2019/1024 relativa a los datos abiertos y la reutilización de la información del sector público).

En términos sustantivos, el principal conflicto al que se enfrentan las administraciones a la hora de decidir si permitir o no el acceso de la comunidad investigadora a los datos administrativos que custodian es el de las implicaciones que esa cesión pueda tener en relación a la salvaguarda de la confidencialidad y la privacidad de los 
individuos. Los datos administrativos no los recopilan las Administraciones públicas con fines investigadores -lo que crea problemas y por defecto, los titulares no han dado consentimiento expreso para que se usen con otros fines-. Esto, en ausencia de un marco legal que explícitamente establezca unas directrices para facilitar el uso de estos datos para la investigación o el análisis, empuja de manera natural a las administraciones a mantener una actitud en exceso recelosa a la hora de facilitar a terceros la explotación de estos datos.

\subsection{La ausencia de expertise en protección de datos en el seno de las Administraciones públicas}

Las restricciones que impone esta legislación garantista se magnifican por un problema más específico: la ausencia, por lo general, de auténticos expertos en materia de protección de datos en el seno de las Administraciones públicas. Ello conduce a una tendencia estructural hacia la realización de una interpretación siempre muy defensiva de la legislación de protección de datos por parte de las instituciones que mantienen las bases de datos administrativas.

Esta falta de expertise en protección de datos, añadido a una razonable y comprensible aversión al riesgo de los funcionarios responsables del mantenimiento de estas bases de datos tienden a desembocar en una socialmente indeseable tendencia a la opacidad. Igual que como dice el dicho, «nadie fue despedido nunca por comprar IBM», nadie se metió nunca en un lío por negarse a la utilización de unos datos para la investigación. Dicho de otra forma, los incentivos que tienen los responsables de estos ficheros son completamente opuestos a su utilización para investigación y en particular a su cesión para ese fin. En los casos en que existe ambigüedad, una interpretación favorable a la cesión de datos para el análisis puede conllevar responsabilidades para el funcionario o directivo público al cargo de los mismos, mientras que cuesta imaginar bajo qué circunstancias este mismo responsable pueda beneficiarse de las eventuales y siempre inciertas consecuencias beneficiosas de la cesión (en términos de mayor conocimiento científico, inputs de mayor calidad de cara a la elaboración de políticas públicas futuras, etcétera).

Este déficit de expertise no consiste solo en ausencia de perfiles profesionales especializados en diferentes disciplinas en protección de datos en el seno de las administraciones, sino que se manifiesta en las estructuras que gestionan estos registros. Al fin y al cabo, los «productores» de registros administrativos son fundamentalmente administraciones encargadas de lo que definimos genéricamente como «gestión». Son los encargados de recaudar los impuestos, gestionar el flujo de cotizaciones y prestaciones de la Seguridad Social, el funcionamiento del sistema sanitario, reducir la accidentalidad vial o asegurar el mantenimiento regular del padrón de población. El ejercicio de estas funciones en las sociedades modernas supone una gran especialización de las administraciones encargadas de llevarlas a cabo, que por lo general las realizan con una gran eficiencia. 
Esta eficiencia y especialización «productiva», sin embargo, puede acabar reñida con la realización de otros fines necesarios para una buena gestión de las bases de datos, especialmente si esas bases han de generarse y mantenerse con los ojos puestos en la evaluación de agentes externos. La difusión de los registros es una tarea a menudo compleja, que conlleva la depuración de los registros, la interpretación y selección de los datos con la finalidad buscada de antemano, la creación de metadatos y la elaboración de documentos metodológicos, casos de uso... Tareas todas muy laboriosas e intensivas en mano de obra especializada ya no tanto en la naturaleza sustantiva de la política o programa en cuestión, sino en tratamiento y gestión de datos. La complejidad de estas se multiplica cuando además la evaluación -y las exigencias que impone a la hora de mantener los registros-no es tenida en cuenta en el diseño original de estos registros, lo que suele ser un problema especialmente en los registros de mayor longevidad.

Una de estas tareas que estas administraciones han de realizar es la de la aplicación de técnicas informáticas de anonimización que constituyen hoy un campo específico dentro de la ciencia de datos. Los avances en inteligencia artificial, que permiten incluso reconstruir datos de registro a partir de datos agregados, hacen que algunos métodos tradicionales para combinar la difusión de información con el mantenimiento del requisito de la anonimización se estén quedando obsoletos. Así, por ejemplo, puede resultar posible reconstruir una matriz original con identificadores por individuo a partir de distribuciones marginales procedentes de esa matriz en la que los identificadores no están presentes. Y las soluciones para mantener la anonimización ante estos problemas no son inocuas para el análisis (véase por ejemplo AEPD, 2019). Todo ello hace aún más problemático la infradotación de científicos de datos especializados en la gestión de estas bases administrativas.

En definitiva, todas estas tareas difícilmente pueden ser llevadas a cabo con eficacia en ausencia de recursos humanos y materiales específicos. Y en ausencia de cambios institucionales, no es realista pensar que las distintas administraciones productoras de registros vayan a dedicar parte de sus medios a una tarea que muchas veces no perciben como central dentro de sus funciones.

\subsection{Fragmentación competencial}

La ausencia de concentración de responsabilidades en una sola institución contribuye a veces a magnificar al problema anterior. No existe, a día de hoy, una asignación específica a ningún organismo no ya de las tareas anteriores sino ni siquiera de las funciones de evaluación que en última instancia podrían ejercer una labor tractora sobre la necesidad de impulsar las tareas mencionadas en el punto previo. Desaparecida la Agencia Estatal de Evaluación de las Políticas Públicas y la Calidad de los Servicios (AEVAL), cuyas competencias nunca fueron suficientes para llevar a cabo el cambio cultural e institucional necesario, existen a día de hoy una maraña de entidades con competencias directa o indirectamente relacionadas con la 
evaluación o el diseño de políticas - cabe destacar entre ellas la Autoridad Independiente de Responsabilidad Fiscal (AIReF), que desde 2021 dispone de nueva División de Evaluación del Gasto Público y que gracias a unas amplias competencias formales de acceso a datos en poder de las Administraciones públicas ha sido capaz de generar el embrión de un hub de datos administrativos dedicados a la evaluación- sin que ninguna de ellas disponga de la capacidad suficiente para realizar esa tarea de impulso y coordinación.

Una vertiente de este problema es la existencia de una visión patrimonialista de los registros administrativos por parte de las distintas administraciones responsables. Los titulares de los ficheros adoptan en muchas ocasiones una actitud privativa sobre los datos, oponiéndose explícita o tácitamente a su utilización por otros. Este problema no es fácil, en la práctica, de distinguir del anterior pues el titular del fichero a menudo usa argumentos de carácter legal (de nuevo, valiéndose de la interpretación defensiva de un marco legal interpretable) para avalar su oposición a la cesión de los datos. Y la oposición a la cesión de una institución provoca reacciones de cadena en la misma dirección en las demás.

Evidentemente, la existencia de una pluralidad de administraciones y entidades gestoras de datos administrativos no es siempre un obstáculo: es muy posible que esta pluralidad también facilite el surgimiento y generalización de buenas prácticas. El reto es lograr establecer el marco que posibilite y potencie esta competencia y colaboración virtuosa mientras se minimizan los obstáculos que la fragmentación genera.

\subsection{Debilidad de cultura de la evaluación}

La debilidad de una cultura de la evaluación, entendida de modo genérico como creencia en que las políticas públicas han de estar informadas por la evidencia y que sus efectos han de ser analizados de acuerdo a los objetivos marcados $^{2}$, reduce los incentivos de los gestores y las administraciones a liberar y compartir datos cuando perciben que existe incertidumbre sobre el marco legal que ampara la distribución y explotación de datos.

Una versión especialmente perniciosa de este problema se produce cuando el titular del fichero se resiste a distribuir información no porque no incorpore en su cálculo coste-beneficio la contribución al conocimiento y a la mejora de la política pública que la explotación de datos puede generar, sino porque es intrínsecamente reacio a que se evalúe externamente la gestión de un programa o de una política.

Esta práctica es significativamente más problemática que la anterior, pues si los administradores eligen selectivamente qué ficheros liberar y cuáles no en base a los resultados esperados de la evaluación, ello acabará introduciendo un sesgo en los resultados de las evaluaciones que se realicen a partir de los datos cedidos.

${ }^{2}$ Un buen estudio empírico que aspira a medir la presencia de esta «cultura de la evaluación» a través de una encuesta a decisores públicos se puede encontrar en AIReF (2021). 
Sin embargo, es importante señalar que el rechazo a liberar datos por las consecuencias de la evaluación puede tener otras muchas causas. Una posibilidad es que los gestores hayan desarrollado un interés intrínseco en el mantenimiento de una política o medida concreta, cuyo futuro pueda verse comprometido por una evaluación de los efectos de esa política. En este caso, el sesgo que esa actitud introduciría en las evaluaciones sería evidente. Pero también puede ser que un motivo por el cual las administraciones se opongan a liberar datos para la evaluación, más pedestre pero quizá más común, sea que el acceso por parte de terceros a esos registros puede revelar imperfecciones, errores, o sencillamente rutinas en la gestión de esos ficheros (a veces causado por la falta de recursos y de profesionales especializados en la gestión de estos ficheros), que los administradores prefieran mantener «fuera del radar».

Esto en el fondo revela una ventaja ulterior de la extensión de la cultura de la evaluación: la expectativa de que los datos almacenados en registros administrativos serán en algún momento analizados por evaluadores externos hará que los responsables de esos ficheros inviertan en mantenerlos en buen estado. Pero también muestra lo crucial que resulta la regulación y el ejercicio de las políticas de protección de datos: un acceso a los datos poco garantista debilitará los incentivos de los gestores a mantener datos con información rica, en la medida que la difusión indeseada de esa información pueda tener consecuencias comprometedoras para ellos en el futuro.

\section{Medidas}

\subsection{Un marco legal proclive a la cesión de datos que dé seguridad jurídica a las administraciones}

Como se ha señalado en la sección anterior, una buena parte de la reticencia de las administraciones a la cesión y uso de sus datos para usos de investigación o evaluación emana de la percepción que estos administradores tienen de la ausencia de amparo legal para avalar las decisiones de cesión de datos a terceros. Es, por consecuente, importante acomodar el marco legal que regula la gestión de los datos administrativos para que se incorpore de manera explícita este nuevo uso, con el fin de que las administraciones tengan un amparo claro y transparente para poder ceder datos, y que conozcan con claridad bajo qué condiciones de acceso pueden ceder estos ficheros.

Desde el Ministerio de Inclusión, Seguridad Social y Migraciones hemos promovido cambios legales en este sentido para permitir que los datos de la Seguridad Social, con las debidas y necesarias garantías, puedan ser utilizados por la comunidad investigadora. Así, el nuevo artículo 77 de la Ley General de Seguridad Social (modificado en el Real Decreto Ley 2/2021), que define explícitamente los fines para los cuales podrán ser usados los datos obtenidos por la Administración de la Seguridad Social en el ejercicio de sus funciones, incluye, por primera vez, en su apartado $\mathrm{m}$ ): 
«fines de investigación científica en el ámbito de la protección social, en el marco establecido por el Reglamento (UE) 2016/679 del Parlamento Europeo y del Consejo de 27 de abril de 2016 relativo a la protección de las personas físicas en lo que respecta al tratamiento de datos personales y a la libre circulación de estos datos (Reglamento general de protección de datos), incluidas las posibles comunicaciones instrumentales que, a efectos de la realización de la investigación, resulte preciso efectuar a sujetos distintos de aquellos que lleven a cabo directamente dicha investigación. Se entenderán comprendidas en esta finalidad las actividades de evaluación de las políticas públicas en materia de protección social.

Los tratamientos que se efectúen en relación con esta finalidad se limitarán a los datos estrictamente imprescindibles para la realización de la actividad de que se trate, utilizándose los procedimientos adecuados que no permitan la identificación de los interesados. Ello no impedirá la comunicación de datos sin anonimizar a efectos meramente instrumentales cuando ello resulte imprescindible para realizar la actividad, se limite a los datos estrictamente necesarios, se garantice que el encargado del tratamiento no podrá utilizarlos con otra finalidad y el tratamiento ulterior garantice la no identificación de los interesados.»

Por supuesto, esto es solo un avance parcial, que afecta a solo uno de los proveedores de bases administrativas. Pero creemos que es un paso importante. Si se logra consolidar el hacer explícito en la regulación de gestión de datos la necesidad de facilitar su uso para la investigación y la evaluación, no solo se construirá un marco legal que dé certidumbre a los gestores y se haga más fácil y más seguro la explotación de estos datos para estos fines, sino que también se facilitará la movilización de recursos para eliminar los demás obstáculos identificados en la sección anterior.

En un plano más general, y de cara a la universalización de estas prácticas, las recomendaciones de cambios normativos señalados en AIReF (2020) para la creación de una institucionalización de estas prácticas son, creemos, el necesario punto de partida.

\subsection{Creación de nuevas infraestructuras estables para la cesión y explotación de datos}

En primer lugar, un cambio necesario es la incorporación de más perfiles especialistas en gestión y difusión de datos en el seno de las instituciones que gestionen datos administrativos. Las nuevas exigencias de la cesión para uso investigador y de evaluación de políticas exige de los administradores de estas bases de datos unas skills y una especialización diferente a las tradicionales, más centradas en los usos internos y de gestión que tradicionalmente se han dado a estos datos. 
Pero más allá de este cambio en los perfiles, creemos que es necesario también cambiar las infraestructuras que permitan compaginar difusión y explotación con fines de investigación con la necesaria protección de la confidencialidad de los datos. En la Seguridad Social, un paso que hemos dado ha sido la puesta en marcha de «salas seguras» en el seno de instalaciones propias (hasta la fecha tres, situadas en diferentes partes del territorio) que permitan a investigadores externos, con las debidas garantías, el acceso a datos internos que por su naturaleza no puedan ser difundidos.

Las salas seguras son una forma de permitir el acceso a datos identificables (y por tanto muy sensibles) a cambio de un fuerte mecanismo de protección de esos datos. Son un paso importante, pero para maximizar el impacto de la explotación de estos datos es necesario poder cruzar ficheros procedentes de diferentes administraciones. Como el proceso de cruce introduce todavía mayores vulnerabilidades en términos de privacidad y confidencialidad, es importante acompañar ese proceso con la creación de infraestructuras que permitan combinar accesibilidad para la investigación con procesos más complejos y garantistas de anonimización de los datos.

La buena noticia es que las tecnologías para combinar protección de la anonimización con el cruce de datos procedentes de diferentes instituciones existen. En AIReF (2020) se hace un repaso de ellas. En Francia, por ejemplo, el Centro de Acceso Seguro a Datos (CASD) es un organismo creado a partir del Instituto Nacional de Estadística (INSEE). Este organismo actúa como «tercero seguro» frente a las distintas administraciones, que seguirían sin tener acceso a los identificadores de los registros de las demás, y que se encargaría de unir ficheros a través de identificadores y «devolverlos» de forma anonimizada a sus usuarios finales. Esta institución tampoco realiza investigación propia, para evitar un potencial conflicto de intereses. Igualmente, la decisión de facilitar el acceso a un determinado conjunto de datos se hace en función de peticiones concretas, que son evaluadas por un comité diferenciado que no se encarga de las tareas de cesión y depuración de los ficheros.

Para avanzar en esa dirección, el camino más razonable debería ser el de profundizar en el grupo de trabajo estable entre los principales productores de datos en España: el Instituto Nacional de Estadística, la Agencia Tributaria, el Servicio Público de Empleo Estatal, y la Seguridad Social, y que podría ser el germen de esa eventual nueva estructura. Este nuevo «consorcio» estable debería también contar con la participación de la Agencia Española de Protección de Datos (AEDP), que actuaría como garante último de que los procesos de explotación sean totalmente compatibles con el mantenimiento de la confidencialidad y anonimidad de los datos.

Esta institucionalización de la colaboración inter-institucional también tendría otras consecuencias saludables, al suponer un impulso a la estandarización de criterios para identificar individuos u otras unidades de análisis (empresas, entidades, hogares...) en diferentes bases de datos, compartir el expertise generado dentro de cada una de estas instituciones de manera transversal entre ellas, y el aprovechar las economías de escala que se generarían por cada fusión de datos, al ser estas fusiones explotables por investigadores diferentes a los que hicieron la petición original. 


\section{Conclusiones}

En última instancia, el éxito del proceso de apertura de datos administrativos para la investigación y el análisis dependerá de si estas u otras intervenciones acaban generando una fuerte cultura de la evaluación y del análisis en la comunidad académica e investigadora, pero también con capacidad de permear el día a día de las Administraciones públicas.

España parte con algo de desventaja en la explotación de las bases de datos administrativas para mejorar la elaboración de políticas públicas, pero creemos que la toma de conciencia de los obstáculos, las iniciativas que se están desplegando, el aprendizaje a partir de la experiencia reciente de los países en nuestro entorno, y la cada vez mayor concienciación tanto en la sociedad como en las instituciones acerca del gran potencial que estos cambios pueden generar para el bienestar común son motivos para ser razonablemente optimistas.

\section{Referencias bibliográficas}

AEPD, Agencia Española de Protección de Datos. (2019). La k-anonimidad como medida de la privacidad. Nota técnica 0/2019.

https://www.aepd.es/sites/default/files/2019-09/nota-tecnica-kanonimidad.pdf

AIReF, Autoridad Independiente de Responsabilidad Fiscal. (2020, octubre). Opinión para una estrategia de acceso a datos administrativos. Opinión 1/2020.

https://www.airef.es/wp-content/uploads/2020/10/OPINION_DATOS/201005.-

Opinion-Acceso-Datos.pdf

AIReF, Autoridad Independiente de Responsabilidad Fiscal. (2021). La institucionalización de la evaluación de políticas públicas en Castilla y León: situación actual y propuestas. Estudio de Evaluación de Políticas.

https://www.airef.es/wp-content/uploads/2021/10/ESTUDIOS/PDF-WEBaccesible-CASTILLA-Y-LEON-1.pdf

Almunia, M., \& Rey-Biel, P. (2020). Por un cambio de cultura en la gestión de los datos en España: Una propuesta de reforma. EsadeEcPol Insight, 17/2020. https://www.airef.es/wp-content/uploads/2020/10/OPINION_DATOS/201005 .Opinion-Acceso-Datos.pdf

Brugarolas, P., De la Rica, S., \& Gorjón, L. (2020). El uso de datos administrativos para la investigación. El caso de Dinamarca: un ejemplo de buenas prácticas. Fundación ISEAK. https://iseak.eu/wp-content/uploads/2020/11/El-sistema-danés-demicrodatos_DFG.pdf

De la Fuente, A., de Rus, G., Fernández M., García M. A., Jansen M., Jiménez S., Novales A., Onrubia J., Pérez Renovales J., Sastre E., \& Sicilia J. La evaluación de políticas públicas en España: antecedentes, situación actual y propuestas para una reforma. FEDEA Policy Papers 2021/09.

https://documentos.fedea.net/pubs/fpp/2021/10/FPP2021-09.pdf?utm_source= wordpress\&utm_medium=portada\&utm_campaign=estudio 
Directiva (UE) 2019/1024 del Parlamento Europeo y del Consejo de 20 de junio de 2019 relativa a los datos abiertos y la reutilización de la información del sector público (versión refundida). Diario Oficial de la Unión Europea. http://data.europa.eu/eli/dir/2019/1024/oj

Real Decreto-ley $2 / 2021$, de 26 de enero, de refuerzo y consolidación de medidas sociales en defensa del empleo. Boletín Oficial del Estado, n. ${ }^{\circ}$ 23, de 27 de enero de 2021. https://www.boe.es/eli/es/rdl/2021/01/26/2/con

Reglamento (UE) 2016/679 del Parlamento europeo y del Consejo de 27 de abril de 2016 relativo a la protección de las personas físicas en lo que respecta al tratamiento de datos personales y a la libre circulación de estos datos y por el que se deroga la Directiva 95/46/ CE (Reglamento general de protección de datos). Diario Oficial de la Unión Europea. http://data.europa.eu/eli/reg/2016/679/oj

Perrin, S., Barrigar J., \& Gellman R. (2015). Government Information Sharing Is Data Going Out of the Silos, Into the Mines?

https://www.oipc.ab.ca/media/389571/Report_Government_Information_Sharing_ Jan2015.pdf 